at 2 months following discharge. Following a week of fever, vomiting, and diarrhea with dehydration, she developed status epilepticus. A postinfectious communicating hydrocephalus was treated with ventriculoperitoneal shunt. Two months after discharge, she gradually exhibited a behavioral change characterized by aggression toward family members and pets, uncontrolled screaming and crying, inability to sustain attention span, hyperactivity, and decreased language output. Persistent behavior problems were treated with clonidine and Concerta, and were complicated at 4 and $1 / 2$ years of age by hypersexuality (frequent masturbation), hyperorality, and significant weight gain. MRI at age 4 showed diffuse brain atrophy with maximal temporal lobe involvement and at 9 years, decreased hippocampal volume bilaterally. Complex partial seizures at 6 years of age were partially controlled with carbamazepine and lamotrigine. Except for tactile exploratory behavior and clinging to the examiner, the neurological exam was normal. EEG showed focal slowing bilaterally with epileptiform discharges over right and left midtemporal regions. Serial neuropsychological evaluations at 5,7 , and 10 years of age demonstrated expressive and receptive language impairment and an amnestic syndrome. She had poor working memory and verbal learning performance and was unable to make new declarative memories. Her reading, spelling, and arithmetic performance decreased from low average/average to borderline at age 10 years. Oral reading fluency, comprehension, and written expression were in deficient range. (Cohen MJ, Park YD, Kim H, Pillai JJ. Long-term neuropsychological follow-up of a child with Kluver-Bucy syndrome. Epilepsy Behav Dec 2010;19:643-646). (Respond: Dr Morris J Cohen, Medical College of Georgia, $112015^{\text {th }}$ Street, Bt-2601, Augusta, GA 30912).

COMMENT. Kluver and Bucy at the University of Illinois, Chicago, in 1937 and 1938, described a "psychic blindness" and other symptoms following bilateral temporal lobectomy in rhesus monkeys. Terzian et al reported the first case of KB syndrome in man in 1955, following bilateral removal of temporal lobes for intractable epilepsy. In adults, the characteristic symptoms of $\mathrm{KB}$ syndrome include 1) visual agnosia, inability to recognize objects without loss of visual discrimination; 2) hyperorality, urge to examine objects by mouth; 3) hypermetamorphosis, excessive attentiveness/touching of visual stimuli; 4) placidity, loss of normal fear or anger; 5) hypersexuality; and 6) changes in dietary habits. Also, aphasia, memory loss, and seizures occur in human cases. The syndrome is rare, only 27 pediatric cases reported, and is usually incomplete. Hyperorality and hypersexuality are most frequent features, followed by bulimia. Causes in children are various, including herpes simplex encephalitis, HIE, traumatic brain injury, neurocysticercosis, mycoplasma infection, bilateral arachnoid cysts, and encephalopathy.

\title{
PREDICTORS OF MENINGITIS WITH FIRST FEBRILE SEIZURE
}

Researchers in Delhi, India, investigated the prevalence of bacterial meningitis in 497 children aged 6-18 months admitted to hospital with a first seizure with fever. Lumbar puncture was performed in 199 (40\%) infants; 116 with "simple" seizures and 83 "complex." (The hospital has a policy of LP in all patients with complex febrile seizures). Meningitis was diagnosed in 5 infants $(2.4 \%) ; 1(0.86 \%)$ with simple, and $4(4.81 \%)$ with complex features. Risk factors for meningitis included 1) duration of seizure $>30 \mathrm{~min} ; 2$ ) 
postictal drowsiness; and 3) neurological deficits, the most reliable indicator. The mean age was 12.2 months and 12.9 months in patients with and without meningitis, respectively. CSF findings with meningitis were as follows: mean cell count $73.8 \mathrm{~mm} 3$; protein $92.6 \mathrm{~g} / \mathrm{dl}$; glucose $51.2 \mathrm{~g} / \mathrm{dl}$; all cultures were sterile, probably due to prior antibiotics. Blood cultures showed no bacteremia. In 2 patients with meningitis, cause of fever was not identified. Most common causes of fever in the total cohort were URI infection, gastritis, pneumonia, and otitis media. (Batra P, Gupta S, Gomber S, Saha A. Predictors of meningitis in children presenting with first febrile seizures. Pediatr Neurol January 2011;44:35-39). (Respond: Dr Batra, Gaur Green City, C-1019, Indirapuram, Ghaziabad, Uttar Pradesh, India. E-mail: drprernabatra@yahoo.com).

COMMENT. The authors conclude that lumbar puncture may be indicated in children aged 6-18 months who present with a first seizure with fever, especially if the seizure lasts $>30$ minutes, there is postictal drowsiness. or most specifically, there are neurological deficits. The diagnosis of meningitis was made without identifying an organism in 2 patients, and the organism identified was not specified in the remaining 3 cases. Although the CSF gram stain and culture were considered the gold standard for a diagnosis of meningitis, the diagnosis was based mainly on the CSF cell count, protein, and sugar.

Normative CSF profiles of febrile infants, aged 1 to 90 days, with negative bacterial culture and negative PCR for enteroviral infection, were studied at University of Utah, Salt Lake City. (Byington CL et al. J Pediatr Jan 2011;158:33-37). In 677 infants with atraumatic LP, the mean and median CSF WBC were $4.3 / \mathrm{mm} 3$ and $3.0 / \mathrm{mm} 3$, respectively, with a range from 0 to $12 / \mathrm{mm} 3$. CSF WBC counts were higher in the first month vs months 2 and $3 ; 6.1$ v 3.1 and 3.0, respectively. Protein levels were $75.4 \mathrm{mg} / \mathrm{dl}$ vs 58.9 and 39.2, respectively. $(\mathrm{P}<0.001$ for all). CSF glucose levels were lower in the first month compared to month $3 ; 45.3 \mathrm{mg} / \mathrm{dl}$ vs $57.7 \mathrm{mg} / \mathrm{dl}(\mathrm{P}<0.001)$. Normal CSF WBC counts in febrile infants 1 to 90 days are lower than reported in pediatric references.

\section{FEVER-INDUCED EPILEPTIC ENCEPHALOPATHY (FIRES)}

Clinical and imaging characteristics of fever-induced refractory epileptic encephalopathy (FIRES), idiopathic hemiconvulsion-hemiplegia syndrome (IHHS), and related acute encephalopathies are reviewed by researchers at Descartes University, Paris, France; Institute for Pharmacological Research, Milan, Italy: and Institute of Biomedical Imaging, Orsay, France. FIRES begins in school-age children with status epilepticus lasting several weeks, involving perisylvian and mesial temporal structures, and is followed by refractory epilepsy and major cognitive deterioration. IHHS begins in infancy with unilateral clonic status epilepticus and is followed by hemiplegia with refractory epilepsy. The etiology is unknown, but both FIRES and IHHS are acute encephalopathies with inflammation-mediated status epilepticus. The ketogenic diet is recommended to treat both seizures and inflammation. (Nabbout R, Vezzani A, Dulac O, Chiron C. Acute encephalopathy with inflammation-mediated status epilepticus. Lancet Neurol Jan 2011;10:99-108).Respond: Dr R Nabbout. E-mail: rimanabbout@yahoo.com 